\title{
Novedades corológicas para la flora briofítica ibérica. VIII
}

\author{
María Jesús Cano', Juan Guerra1 ${ }^{1}$, Baltasar Cabezudo² \& Rafael Tamajón ${ }^{3}$ \\ 1 Departamento de Biología Vegetal, Área de Botánica, Facultad de Biología, Universidad de Murcia, 30100 Murcia. \\ 2 Departamento de Biología Vegetal (Botánica), Facultad de Ciencias, Universidad de Málaga, 29080 Málaga. \\ 3 Avda. 28 de Febrero 1, Bajo n² 28, 14007 Córdoba.
}

Correspondencia

MJ. Cano

E-mail: mcano@um.es

Recibido: 15 agosto 2017

Aceptado: 2 noviembre 2017

Publicado on-line: 12 diciembre 2017

\section{Resumen}

A partir del estudio de muestras recolectadas y revisadas en el marco del Proyecto Flora Briofítica Ibérica (FBI), y el estudio briofítico que estamos realizando en la Sierra de las Nieves (Málaga) aportamos un total de 32 novedades corológicas provinciales para España y dos para Portugal. A destacar el hallazgo de Scorpidium scorpioides, una especie catalogada como amenazada en España, cuya distribución se extiende al alto valle del Ebro y dos nuevas localidades de Stegonia latifolia, una especie considerada como vulnerable.

Palabras clave: Briófitos, Península Ibérica, Proyecto Flora Briofítica Ibérica.

\begin{abstract}
New chorological records for the Iberian Bryophyte Flora. VIII

From the study of samples collected and reviewed under the Flora Briofítica lbérica Project as well as the examination of bryophytes from Sierra de las Nieves (Málaga), we provide 32 new provincial records for Spain and two for Portugal. Special mention deserves the new Iberian locality for Scorpidium scorpioides, an endangered species in Spain, whose distribution range is extended to upper Ebro valley and two new localities for Stegonia latifolia, classified as vulnerable species in Spain.
\end{abstract}

Key words: Bryophytes, Iberian Peninsula, Flora Briofítica Ibérica Project.

\section{Introducción}

Continuamos la publicación de novedades corológicas provinciales encontradas en los estudios florísticos realizados dentro del Proyecto Flora Briofítica Ibérica [http://www.florabriofiticaiberica. com].

En este artículo incluimos los resultados de los trabajos de prospección llevados a cabo en el citado proyecto durante los años 2016 y 2017 , junto a las recolecciones realizadas por el grupo liderado por Baltasar Cabezudo (Universidad de Málaga) en la Sierra de las Nieves y Rafael Tamajón en Huelva, que han dado como resultado el hallazgo 32 novedades corológicas provinciales para España y dos para Portugal.

Las muestras se encuentran depositadas en el herbario de la Universidad de Murcia (MUB) y los especímenes de Málaga en la Universidad de Málaga (MGC), con duplicados en MUB. 


\section{Resultados}

\section{Musgos}

\section{Acaulon fontiquerianum Casas \& Sérgio}

Portugal: Algarve, cabo de San Vicente, $37^{\circ} 1^{\prime} \mathrm{N}$, 859’O, 69 msnm, 21-III-2016, Cano 9411 (MUB 52576).

Especie conocida de unas pocas localidades en la región Mediterránea (Cerdeña, Córcega, España, Francia, Portugal, Sicilia), Islas Canarias y recientemente del oeste de Turquía (Cano et al. 2012, Kirmaci \& Erdağ 2014). En la Península Ibérica se conoce de España y Portugal, en las provincias de Albacete, Almería, Ciudad Real, Gerona, Murcia y Valencia y del Alto Alentejo, Baixo Alentejo y Estremadura respectivamente (Brugués et al. 2016). El espécimen fue recolectado sobre suelo en claro de matorrales y es novedad para el Algarve portugués.

\section{Anacolia menziesii (Turner) Paris}

España: Albacete, Sierra de Alcaraz, Calar de la Sima, Los Palancares, 1.390 msnm, 38²0’N, 02²8'O, 27-VI-2016, Guerra (MUB 55005).

Cano (2010) recoge la existencia de esta especie en Almería, Granada, Soria y Zamora. Por tanto, esta localidad supone una considerable extensión de su área en la Península Ibérica, ya que resulta novedad florística para la provincia de Albacete. Se ha recolectado en una gran población desarrollada sobre rocas calizas y hendiduras con suelo, por lo que no puede considerarse una especie acidófila, como se creía hasta el momento.

\section{Astomum levieri Limpr.}

España: Huelva, Huelva capital, parque Moret, 37º $16^{\prime} \mathrm{N}, 6^{\circ} 56^{\prime} \mathrm{O}, 60 \mathrm{msnm}$, II-2017, Tamajón (MUB 55224).

Especie infrecuente en la Península, que ha sido citada en algunas localidades dispersas de varias provincias españolas y del Algarve en Portugal (Guerra 2006a). Ha sido recolectada en suelos de taludes en claros de matorrales.

\section{Bryoerythrophyllum recurvirostrum (Hedw.) P.C. Chen}

España: Málaga, Ronda, Sierra de las Nieves, cabecera de la Cañada de las Ánimas y Enmedio, $36^{\circ} 41^{\prime} \mathrm{N}, 5^{\circ} 00^{\prime} \mathrm{O}, 1.750 \mathrm{msnm}, 19-\mathrm{V}-2017$, Cabezudo et al. (MUB 55461).
En el sur de la Península se ha citado de Almería, Granada y Jaén ( $c f$. Gallego 2006a). Esta localidad representa una novedad para la provincia de Málaga. Ha sido recolectada en hendiduras de rocas calizas, junto a Bryum capillare Hedw. y Tortella tortuosa (Schrad. ex Hedw.) Limpr.

\section{Climacium dendroides (Hedw.) F. Weber \& D. Mohr}

España: Cantabria, puerto de Riofrío, $43^{\circ} 2^{\prime} \mathrm{N}$, 4²3'O, 1.748 msnm, 18-VII-2016, Cano 9472 (MUB 55107); pr. Corconte, 432'N, $3^{\circ} 55^{\prime} \mathrm{O}, 840$ msnm, 1-X-2017, Cano 10233 (MUB 55706).

Distribuida en el norte y centro de la Península Ibérica, asociada en general a suelos higroturbosos $\mathrm{y}$ pastizales húmedos cercanos a arroyos en zonas montañosas. Esta especie no se conocía en Cantabria, aunque sí de provincias próximas como Asturias o León (Fuertes \& Olivan 2014). Las muestras fueron recolectadas respectivamente en un suelo higroturboso y en un pastizal húmedo.

\section{Cynodontium bruntonii (Sm.) Bruch \& Schimp.}

España: Cuenca, sierra de Valdemeca, Collado Bajo, 40 $10^{\prime} \mathrm{N}, 01^{\circ} 45^{\prime} \mathrm{O}, 1.830 \mathrm{msnm}, 6-\mathrm{V}-2017$, Cano 9939 (MUB 55310).

Aunque se trata de una especie frecuente en la Península Ibérica sobre roquedos silíceos expuestos, no había sido citada hasta ahora de la provincia de Cuenca ( $c f$. Heras \& Infante 2015a). El espécimen fue recolectado sobre un roquedo de arenisca en un pinar de Pinus sylvestris L. y Pinus pinaster Aiton.

\section{Dichodontium pellucidum (With.) R.H. Zander}

España: Cuenca, sierra de Valdemeca, Fuente de la Ardilla, 40 ${ }^{\circ} 10^{\prime} \mathrm{N}, 01^{\circ} 44^{\prime} \mathrm{O}, 1.360 \mathrm{msnm}, 6-\mathrm{V}-$ 2017, Cano 9933 (MUB 55303).

Según Brugués \& Ruiz (2015) se trata de una especie frecuente, sobre todo en el norte de la $\mathrm{Pe}$ nínsula, en taludes y márgenes de cursos de agua tanto en sustrato ácido como básico. No había sido citada en Cuenca, por lo que es novedad para la provincia. La muestra fue encontrada en el borde de un arroyo sobre areniscas, en un pinar de $P$. sylvestris y $P$. pinaster.

\section{Entosthodon pulchellus (H. Philib.) Brugués}

España: Cuenca, Saelices, molino del Castillejo, $39^{\circ} 53^{\prime} \mathrm{N}, 02^{\circ} 49^{\prime} \mathrm{O}, 792$ msnm, 2-IV-2017, Cano 9867 (MUB 50319); Zafrilla, fuente del Berro, 
$40^{\circ} 12^{\prime} \mathrm{N}, 01^{\circ} 32^{\circ} \mathrm{O}, 1.342 \mathrm{msnm}, 7-\mathrm{V}-2017$, Cano 9952 (MUB 55322).

Especie relativamente frecuente en suelos calcáreos en la Península Ibérica, que no había sido citada de Cuenca ( $c f$. Brugués \& Ruiz 2010). Ambos especímenes fueron recolectados en repisas de roquedos calizos.

\section{Ephemerum recurvifolium (Dicks.) Boulay}

España: Sevilla, Carmona, $37^{\circ} 28^{\prime} \mathrm{N}, 5^{\circ} 37^{\prime} \mathrm{O}, 110$ msnm, 19-III-2016, Cano 9397 (MUB 52564).

Según Infante et al. (2010), esta especie se conoce en la Península Ibérica de varias provincias del sur y este peninsular (Alicante, Almería, Castellón, Ciudad Real, Huesca, Valencia y Zaragoza), Baleares (Mallorca) y Algarve portugués. Por tanto, se trata de una novedad para la provincia de Sevilla. La muestra fue encontrada sobre suelo en pastizal cercano a un arroyo.

\section{Eurhynchiastrum diversifolium (Schimp.) J. Guerra}

España: Albacete, Sierra de Alcaraz, Calar de la Sima, Los Palancares, $38^{\circ} 20^{\prime} \mathrm{N}, \quad 02^{\circ} 28^{\prime} \mathrm{O}$, 1.450/1.500 msnm, 27-VI-2016, Guerra (MUB 52423).

Considerada clásicamente una variedad de Eurhynchiastrum pulchellum (Hedw.) Ignatov \& Huttunen, Guerra (2016) elevó este taxón a la categoría de especie en base a sus características morfológicas y comportamiento ecológico. En la Península Ibérica, se conoce de altas montañas calizas del norte (Pirineos, Sistema Ibérico) y del sur (Sierra Nevada, Cazorla). Es novedad para la provincia de Albacete. Ha sido recolectada en su hábitat usual, hendiduras de rocas calizas no húmedas, a elevada altitud.

\section{Homalothecium lutescens (Hedw.) H. Rob.}

España: Orense, Biobra, 46 $28^{\prime} \mathrm{N}, 06^{\circ} 51^{\prime} \mathrm{O}, 667$ msnm, 17-IV-2017, Cano 9880 (MUB 55244).

Especie ampliamente distribuida en la Península Ibérica, pero que hasta ahora no había sido citada de Orense. El espécimen fue recolectado en un roquedo calizo en un encinar.

\section{Hypnum andoi A.J.E. Sm.}

España: Pontevedra, isla de Arosa, $42^{\circ} 51^{\prime} \mathrm{N}$, 852’O, 5 msnm, 30-X-2016, Cano 9600 (MUB 54071).
En Ríos (2016), esta especie no aparece citada de Pontevedra, aunque sí de provincias cercanas como Lugo y Orense. La muestra ha sido recolectada sobre suelo, en un pinar de P. pinaster.

\section{Hypnum vaucheri Lesq.}

España: Málaga, Ronda, Sierra de las Nieves, cabecera de la Cañada de las Ánimas y Enmedio, $36^{\circ} 41^{\prime} \mathrm{N}, 5^{\circ} 00^{\prime} \mathrm{O}, 1.750 \mathrm{msnm}, 19-\mathrm{V}-2017$, Cabezudo et al. (MUB 55331).

Se trata de un taxón relativamente frecuente en altas montañas calizas ( $c f$. Ríos 2016). En el sur de la Península Ibérica, se conoce de Almería (Sierra de María), Granada (Sierra de Baza y Sierra de la Sagra) y Jaén (Sierra de Cazorla), por tanto, la localidad que aquí se aporta supone una importante extensión de su área hacia el oeste peninsular. Ha sido recolectada en hendiduras de rocas calizas junto a Bryum elegans Nees, Encalypta vulgaris Hedw. y Tortella tortuosa var. fragilifolia (Jur.) Limpr.

\section{Lescuraea plicata (Schleich. ex F. Weber \& D. Mohr) Lindb.}

España: Cantabria, Liébana, subida al pico Coriscao, Alto de Valdeoso, $43^{\circ} 05^{\prime} \mathrm{N}, 04^{\circ} 47^{\prime} \mathrm{O}, 2.009$ msnm, 22-VIII-2017, Cano 10211 (MUB 55527).

Se trata de una especie ártico-alpina, que en la Península Ibérica tiene un área de distribución muy restringida. Así, se conoce de algunas localidades de alta montaña de los Pirineos (Lérida, Huesca y Andorra) y de la cordillera Cantábrica en la provincia de Palencia (Brugués \& Ruiz 2016, Cano \& Guerra 2017). Es novedad para Cantabria. La muestra ha sido recolectada en una repisa de roca caliza.

\section{Microbryum floerkeanum (F. Weber \& D. Mohr) Schimp.}

España: Cádiz, pr. Medina Sidonia, El Berrueco, $36^{\circ} 26^{\prime} \mathrm{N}, 6^{\circ} 2^{\prime} \mathrm{O}$, $35 \mathrm{msnm}$, 24-III-2016, Cano 9430 (MUB 52594). Sevilla, Carmona, 37²8'N, 53’ㅇ, 110 msnm, 19-III-2016, Cano 9397 (MUB 52563).

Esta especie ha sido citada de diferentes localidades de España y del Baixo Alentejo en Portugal (Ros \& Werner 2006a). Las muestras representan las primeras citas de la especie en Andalucía, donde son novedad para las provincias de Cádiz y Sevilla. Han sido recolectadas sobre suelo en un pastizal próximo a un arroyo. 


\section{Neckera besseri (Lobarz.) Jur.}

España: Palencia, Valdecebollas, Peñalba, $42^{\circ}$ $57^{\prime} \mathrm{N}, 4^{\circ} 21^{\prime} \mathrm{O}, 1.730 \mathrm{msnm}, 21-\mathrm{VIII}-2016$, Cano 9596a (MUB 55150).

Especie conocida del este de la Península (Alicante, Barcelona, Castellón, Cuenca, Huesca, Lérida, Tarragona y Teruel), Mallorca y de Navarra (Guerra 2010). Se trata de la primera cita en la cordillera Cantábrica. La muestra fue recolectada en una cueva de roca caliza cercana a un arroyo.

\section{Orthothecium intricatum (Hartm.) Schimp.}

España: Cantabria, Liebana, subida al pico Coriscao, Alto de Valdeoso, $43^{\circ} 05^{\prime} \mathrm{N}, 04^{\circ} 47^{\prime} \mathrm{O}, 2.009$ msnm, 22-VIII-2017, Cano 10220 (MUB 55536). Especie conocida del norte y noroeste de la Península Ibérica, que es muy rara en el sur (Casas et al. 2006). Se trata de una novedad para Cantabria. La muestra fue encontrada en una hendidura profunda de un roquedo calizo junto con Myurella julacea (Schwägr.) Schimp. y Platydictya jungermannioides (Brid.) H.A. Crum.

\section{Oxyrrhynchium schleicheri (R. Hedw.) Röll}

España: Málaga, Benahavís, sierra Palmitera, cuenca del río Guadaiza, carril de bajada a La Maquina, $36^{\circ} 33^{\prime} \mathrm{N}, 5^{\circ} 00^{\prime} \mathrm{O}, 420 \mathrm{msnm}, 11-\mathrm{IV}-2017$, Cabezudo et al. (MUB 55232).

La especie no es nada frecuente en la Península Ibérica, donde además se ha confundido con $O x y$ rrhynchium hians (Hedw.) Loeske, mucho más común. Con anterioridad no se había citado en la provincia de Málaga. Suelos relativamente húmedos sobre micasquistos y peridotitas, en alcornocal-pinar.

\section{Philonotis capillaris Lindb.}

España: Málaga, Benahavís, Sierra Palmitera, márgenes del río Guadaiza, cerca del caserío La Maquina, $36^{\circ} 34^{\prime} \mathrm{N}, 5^{\circ} 00^{\prime} \mathrm{O}, 255 \mathrm{msnm}, 11-\mathrm{IV}-$ 2017, Cabezudo et al. (MUB 55293).

Citada en diversas provincias de la Península Ibérica, no se había encontrado en Málaga ( $c f$. Guerra \& Gallego 2010). Recolectada sobre micasquistos en taludes encharcados, muy húmedos y sombríos.

\section{Polytrichum juniperinum Hedw.}

España: Málaga, Tolox, Sierra de las Nieves, vereda del Puerto de los Pilones a Peñón de los Ena- morados, $36^{\circ} 41^{\prime} \mathrm{N}, 5^{\circ} 00^{\prime} \mathrm{O}, 1.750 \mathrm{msnm}, 19-\mathrm{V}-$ 2017, Cabezudo et al. (MUB 55421).

Especie muy común en la Península Ibérica; sin embargo, no había sido citada en la provincia de Málaga (cf. Brugués et al. 2007). Recolectada en pastizales desarrollados sobre suelos calizos muy lavados en el fondo de una dolina.

\section{Polytrichum piliferum Hedw.}

España: Málaga, Benahavís, Sierra Palmitera, El Daidín, cañada de la Cuerna, $36^{\circ} 35^{\prime} \mathrm{N}, 5^{\circ} 01^{\prime} \mathrm{O}$, 610 msnm, 5-V-2017, Cabezudo et al. (MUB 55519).

Se trata de una especie que crece en suelos ácidos, pedregosos y secos, que también puede encontrarse en suelos arenosos calcáreos, muy frecuente en toda la Península ( $c f$. Brugués et al. 2007). En Málaga, donde se cita aquí por primera vez, abundan los terrenos calizos, por lo que es infrecuente o rara. Ha sido recolectada en taludes secos en un alcornocal.

\section{Plagiothecium denticulatum (Hedw.) Schimp.}

España: Cuenca, sierra de Valdemeca, Collado Bajo, $40^{\circ} 10^{\prime} \mathrm{N}, 01^{\circ} 45^{\prime} \mathrm{O}, 1830 \mathrm{mmsn}, 6-\mathrm{V}-2017$, Cano 9938 (MUB 55307); ibidem $1.689 \mathrm{msnm}$, Cano 9944 (MUB 55315).

Especie distribuida en la Península Ibérica mayoritariamente en la mitad norte y en Sierra Nevada (Casas et al. 2006). Los especímenes son novedad para la provincia de Cuenca y han sido recolectados en hendiduras de areniscas.

\section{Protobryum bryoides (Dicks.) J. Guerra \& M.J. Cano}

España: Ciudad Real, Santa Cruz de Mudela, Casa

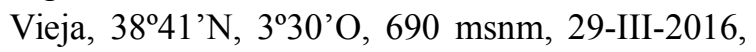
Cano 9439, MUB (52614). Cuenca, Saelices, molino del Castillejo, $39^{\circ} 53^{\prime} \mathrm{N}, 02^{\circ} 49^{\prime} \mathrm{O}, 792 \mathrm{msnm}$, 2-IV-2017, Cano 9864 (MUB 55217).

Especie relativamente frecuente en la Península Ibérica, que no se había encontrado en las provincias de Ciudad Real y Cuenca ( $c f$. Guerra 2006b). La muestra de Ciudad Real fue encontrada en un suelo descubierto y la de Cuenca en un muro con suelo acumulado.

\section{Pseudotaxiphyllum elegans (Brid.) Z. Iwats.}

España: Cuenca, sierra de Valdemeca, Collado Bajo, $40^{\circ} 10^{\prime} \mathrm{N}, 01^{\circ} 45^{\prime} \mathrm{O}, 1830 \mathrm{msnm}, 6-\mathrm{V}-2017$, Cano 9938 (MUB 55309). 
Especie distribuida principalmente en bases de troncos y roquedos silíceos de áreas montañosas del oeste y norte de la Península Ibérica (Casas et al. 2006). No se conocía de Cuenca, por lo que la cita representa una novedad para la provincia. $\mathrm{La}$ muestra fue recolectada en una hendidura profunda de un roquedo de arenisca, en pinar de $P$. sylvestris y $P$. pinaster.

\section{Rhabdoweisia fugax (Hedw.) Bruch \& Schimp.}

Portugal: Beira Baixa, Penhas da Saúde, $40^{\circ} 17^{\prime} \mathrm{N}$, 7³2’O, 1291 msnm, 19-IV-2017, Cano 9911 (MUB 55275).

Especie conocida del norte, centro y oeste peninsular, generalmente en fisuras de roquedos silíceos. En Heras \& Infante (2015b) este taxón no se cita de Beira Baixa. El espécimen se encontraba en una hendidura de roca granítica.

\section{Scleropodium cespitans (Müll. Hal.) L.F. Koch}

España: Cuenca, sierra de Valdemeca, Fuente de la Ardilla, $40^{\circ} 10^{\prime} \mathrm{N}, 01^{\circ} 44^{\prime} \mathrm{O}, 1360 \mathrm{msnm}, 6-\mathrm{V}-$ 2017, Cano 9932 (MUB 55302).

Especie poco frecuente, confinada principalmente al noroeste de la Península (Casas et al. 2006). Se trata de una novedad para la provincia de Cuenca. La muestra fue encontrada en el borde de un arroyo sobre areniscas en un pinar de $P$. sylvestris y $P$. pinaster.

\section{Scorpidium scorpioides (Hedw.) Limpr.}

España: Burgos, Valle de Valdebezana, Cabañas de Virtus, $43^{\circ} 0$ ' N, $03^{\circ} 51^{\prime} \mathrm{O}, 840 \mathrm{msnm}, 26-\mathrm{VIII}-$ 2017, Cano 10223 (MUB 55515).

Se trata de una especie rara en la Península Ibérica, que ha sido incluida en la categoría de especies "Amenazadas" (En) en la última lista roja de los briófitos de España (Brugués \& GonzálezMancebo 2012). En la revisión del género realizado en la Península Ibérica, Fuertes et al. (2005) sólo la citan de dos áreas disyuntas: una en los Pirineos (Andorra y Lérida) y otra en el Sistema Ibérico (Aranda de Moncayo), donde no ha vuelto a ser encontrada. Por tanto, esta cita amplía la distribución de la especie al alto valle del Ebro. El espécimen fue recolectado en una turbera, embebido en el agua.

\section{Stegonia latifolia (Schwägr.) Venturi ex Broth.}

España: Asturias, Saliencia, alto de la Farrapona, $43^{\circ} 5^{\prime} \mathrm{N}, 6^{\circ} 5^{\prime} \mathrm{O}, 1815 \mathrm{msnm}, 16-\mathrm{VII}-2016$, Cano 9462 (MUB 55097). Burgos, subida al macizo de Castro Valnera, $43^{\circ} 8^{\prime} \mathrm{N}, 3^{\circ} 40^{\prime} \mathrm{O}, 1445 \mathrm{msnm}, 17$ VIII-2016, Cano 9570 (MUB 55126).

Especie poco frecuente en la Península Ibérica, conocida preferentemente de roquedos calizos en zonas de alta montaña. Hasta el momento se conocía de unas pocas localidades en Pirineos (Andorra y Lérida) y de una localidad en la cordillera Cantábrica (Asturias, Peña Ubiña) (Ros \& Werner 2006b). Así, ha sido incluida en la categoría de especies "Vulnerables" ( $\mathrm{Vu}$ ) en la última lista roja de los briófitos de España (Brugués \& GonzálezMancebo 2012). Aquí se aportan dos nuevas localidades cantábricas de la especie. Una de ellas es novedad para Burgos. La cita asturiana sería hasta el momento la más occidental de la Península. Ambos especímenes fueron recolectados en fisura de roca caliza.

\section{Syntrichia minor (Bizot) M.T. Gallego, J. Guerra, M.J. Cano, Ros \& Sánchez-Moya}

España: Almería, Sierra de María, ca. Vélez Rubio, $37^{\circ} 68^{\prime} \mathrm{N}, 2^{\circ} 24^{\prime} \mathrm{O}, 1.200 \mathrm{msnm}$, XI-2000, Guerra et al. (MUB 52936).

En la Península Ibérica, se conocía de las provincias de Cádiz y Jaén ( $c f$. Gallego 2006b), por lo que esta localidad representa una notable expansión de su área. Se ha recolectado en repisas de rocas calizas con suelo acumulado.

\section{Syntrichia latifolia (Bruch ex Hartm.) Huebener}

España: Badajoz, Alburqueque, río Gévora, molino las Lobas, $39^{\circ} 11^{\prime} \mathrm{N}, 7^{\circ} 4^{\prime} \mathrm{O}, 254 \mathrm{msnm}, 20-\mathrm{IV}$ 2017, Cano 9926 (MUB 55289).

Citada en diversas provincias de la Península Ibérica, no se había encontrado en Badajoz ( $c f$. Gallego 2006b). La muestra fue recolectada entre las raíces y base de Alnus glutinosa (L.) Gaertn.

\section{Tortula schimperi M.J. Cano, O. Werner \& J. Guerra}

España: Gerona, Cerdanya, Tossa d'Alp, 42 ${ }^{\circ} 19^{\prime} \mathrm{N}$, 01 ${ }^{\circ} 53$ 'E, 2439 msnm, 13-VII-2017, Cano 10126 (MUB 55540).

Se conoce de diferentes localidades del tercio septentrional y centro de la Península Ibérica (Cano 2006, De Antonio \& Lara 2007, Guerra et al. 2008). Se trata de una novedad para la provincia de Gerona. La muestra fue recolectada en una fi- 
sura de roca ácida.

\section{Hepáticas}

\section{Riccia crystallina $\mathbf{L}$.}

España: Murcia, San Pedro del Pinatar, cerca de la playa de la Torre Derribada, 37 $50^{\circ} \mathrm{N}, 0^{\circ} 45^{\prime} \mathrm{O}, 2$ m, 26-III-2017, Cano 9862 (MUB 55215).

Especie frecuente en suelos arenosos, principalmente en cotas bajas, de la mitad sur de la Península y Baleares, que es más rara hacia el norte (Casas et al. 2009). Esta especie no se cita en el catálogo de briófitos de Murcia (Cano et al. 2010), por lo que supone una novedad para esta provincia. El espécimen se recolectó en un suelo arenoso entre matorrales.

\section{Agradecimientos}

A María Teresa Gallego (Universidad de Murcia) por la identificación de Syntrichia minor. Trabajo realizado en el marco del Proyecto Flora Briofítica Ibérica, subvencionado por el MINECO (CGL2015-64068P) y FEDER. Las muestras de la Sierra de las Nieves (Málaga) fueron colectadas gracias al convenio de colaboración suscrito por la Consejería de Medio Ambiente y Ordenación del Territorio de la Junta de Andalucía y la Universidad de Málaga.

\section{Referencias}

Brugués M, Ruiz E \& Casas C. 2007. Polytrichaceae. En Flora Briofítica Ibérica, vol. I (Brugués M, Ros RM \& Guerra J, eds.). Murcia: Universidad de Murcia \& Sociedad Española de Briología, pp. 10-128.

Brugués M \& Ruiz E. 2010. Entosthodon. En Flora Briofítica Ibérica, vol. IV (Guerra J, Brugués M, Cano MJ \& Cros RM, eds.). Murcia: Universidad de Murcia \& Sociedad Española de Briología, pp. 44-59.

Brugués M \& González-Mancebo JM. 2012. Lista roja de los briófitos amenazados de España. En Atlas y libro rojo de los briófitos amenazados de España (Garilleti R \& Albertos B, coords.). Madrid: Organismo Autónomo Parques Nacionales, pp. 2542.

Brugués M \& Ruiz E. 2015. Dichodontium. En Flora Briofítica Ibérica, vol. II (Brugués $M$ \& Guerra J, eds.). Murcia: Universidad de Murcia \& Sociedad Española de Briología, pp. 56-60.

Brugués M, Cros RM, Sérgio C, Cano MJ \& Guerra J. 2016. Acaulon fontiquerianum. En Cartografia de Briòfits. Península Ibèrica i Illes Balears (Brugués $M$, Cros $M$ \& Sérgio $C$ ). Disponible en: http://briofits.iec.cat (accedido el 7-9-2017).

Brugués M \& Ruiz E. 2016. Hypnales: Fabroniaceae,
Leskeaceae, Pterigynandraceae, Thuidiaceae. En Flora Briofítica Ibérica (Fascículos) (Guerra J \& Brugués M, coord.). Murcia: Sociedad Española de Briología, pp. 11-27.

Cano MJ. 2006. Tortula. En Flora Briofítica Ibérica, vol. III (Guerra J, Cano MJ \& Ros RM, eds.). Murcia: Universidad de Murcia \& Sociedad Española de Briología, pp. 146-176.

Cano MJ. 2010. Anacolia. En Flora Briofítica Ibérica, vol. IV (Guerra J, Brugués M, Cano MJ \& Cros RM, eds.). Murcia: Universidad de Murcia \& Sociedad Española de Briología, pp. 270-274.

Cano MJ, Guerra J, Jiménez JA, Gallego MT \& Orgaz JD. 2010. An updated bryophytes check-list of the region of Murcia (southeastern Spain). Anales de Biología 32: 101-131.

Cano MJ, Dirkse G, González-Mancebo JM, Guerra J \& Patiño J. 2012. Acaulon fontiquerianum Casas. En Atlas y libro rojo de los briófitos amenazados de España (Garilleti R \& Albertos B, coords.). Madrid: Organismo Autónomo Parques Nacionales, pp. 167169.

Cano MJ \& Guerra J. 2017. Lescuraea plicata (Schleich. ex F.Weber \& D.Mohr) Broth. En New national and regional bryophyte records 52 . Journal of Bryology 39: 292.

Casas C, Brugués M, Cros RM \& Sérgio C. 2006. Handbook of mosses of the Iberian Peninsula and the Balearic Islands: illustrated keys to genera and species. Barcelona: Institut d'Estudis Catalans.

Casas $C$, Brugués $M$, Cros RM, Sérgio $C$ \& Infante $M$. 2009. Handbook of liverworts and hornworts of the Iberian Peninsula and the Balearic Islands: illustrated keys to genera and species. Barcelona: Institut d'Estudis Catalans.

De Antonio F \& Lara F. 2007. Tortula schimperi M. J. Cano, O. Werner \& J. Guerra, una especie común en los Montes de Valsaín (Segovia). Boletín de la Sociedad Española de Briología 30/31: 53-55.

Fuertes E, Acón M \& Oliván G. 2005. Hamatocaulis y Scorpidium (Calliergonaceae, Bryopsida) en la Península Ibérica. Lazaroa 26: 516.

Fuertes E \& Oliván G. 2014. Climaciaceae. En Flora Briofítica Ibérica, vol. V (Guerra J, Cano MJ \& Brugues M, eds.). Murcia: Universidad de Murcia \& Sociedad Española de Briología, pp. 165-168.

Gallego MT. 2006a. Bryerythrophyllum. En Flora Briofítica Ibérica, vol. III (Guerra J, Cano MJ \& Ros RM, eds.). Murcia: Universidad de Murcia \& Sociedad Española de Briología, pp. 106-113.

Gallego MT. 2006b. Syntrichia. En Flora Briofítica Ibérica, vol. III (Guerra J, Cano MJ \& Ros RM, eds.). Murcia: Universidad de Murcia \& Sociedad Española de Briología, pp. 120-143.

Guerra J. 2006a. Astomum. En Flora Briofítica Ibérica, vol. III (Guerra J, Cano MJ \& Ros RM, eds.). Murcia: Universidad de Murcia \& Sociedad Española de Briología, pp. 196-197.

Guerra J. 2006b. Protobryum. En Flora Briofítica Ibérica, vol. III (Guerra J, Cano MJ \& Ros RM, eds.). Murcia: Universidad de Murcia \& Sociedad Española de Briología, pp. 194-196.

Guerra J. 2010. Neckera. En Flora Briofítica Ibérica, vol. 
V (Guerra J, Cano MJ \& Brugués M, eds.). Murcia: Universidad de Murcia \& Sociedad Española de Briología, pp. 207-217.

Guerra J. 2016. Notes on the genus Eurhynchiastrum (Bryophyta, Brachytheciaceae) in the Iberian Peninsula. Nova Hedwigia 102: 359-365.

Guerra J, Cano MJ, Jiménez JA, Gallego MT \& Orgaz JD. 2008. Novedades corológicas para la flora briofítica ibérica. III. Anales de Biología 30: 61-62.

Guerra J \& Gallego MT. 2010. Philonotis. En Flora Briofítica Ibérica, vol. IV (Guerra J, Brugués M, Cano MJ \& Cros RM, eds.). Murcia: Universidad de Murcia \& Sociedad Española de Briología, pp. 256-269.

Heras P. \& Infante M. 2015a. Cynodontium. En Flora Briofítica Ibérica, vol. II (Brugués $M$ \& Guerra J, eds.). Murcia: Universidad de Murcia \& Sociedad Española de Briología, pp. 76-85.

Heras P. \& Infante M. 2015b. Rhabdoweisia. En Flora Briofítica Ibérica, vol. II (Brugués $M$ \& Guerra J, eds.). Murcia: Universidad de Murcia \& Sociedad Española de Briología, pp. 85-88.
Infante M, Sérgio C \& Heras P. 2010. Ephemeraceae. En Flora Briofítica Ibérica, vol. IV (Guerra J, Brugués M, Cano MJ \& Cros RM, eds.). Murcia: Universidad de Murcia \& Sociedad Española de Briología, pp. 15-25.

Kırmaci M \& Erdağ A. 2014. Acaulon fontiquerianum (Pottiaceae), a new species to the bryophyte flora of Turkey and SW Asia. Polish Botanical Journal 59(2): 229-233.

Ríos D. 2016. Revisión taxonómica del género Hypnum Hedw. (Bryophyta, Hypnaceae) en la Península Ibérica, Islas Baleares y Macaronesia. Tesis Doctoral: Universidad de Murcia.

Ros RM \& Werner O. 2006a. Microbryum. En Flora Briofítica Ibérica, vol. III (Guerra J, Cano MJ \& Ros RM, eds.). Murcia: Universidad de Murcia \& Sociedad Española de Briología, pp. 197-208.

Ros RM \& Werner O. 2006b. Stegonia. En Flora Briofítica Ibérica, vol. III (Guerra J, Cano MJ \& Ros RM, eds.). Murcia: Universidad de Murcia \& Sociedad Española de Briología, pp. 180-183. 